\title{
T-cell acute lymphoblastic leukemia in children: the experience of the treatment protocol ALL-MB-2008 in Uzbekistan
}

\section{Bakhramov Saidjalol Makhmudovich, Ibragimova Sapura Zakhidovna}

Tashkent State Institute of advanced training of doctors

The Scientific Research Institute of Hematology and Blood Transfusion

\section{Email address:}

sapura.71@mail.ru (Ibragimova Sapura Zakhidovna)

To cite this article:

Bakhramov Saidjalol Makhmudovich, Ibragimova Sapura Zakhidovna. T-cell acute lymphoblastic leukemia in children: the experience of the treatment protocol ALL-MB-2008 in Uzbekistan. Journal of research in health science. Vol. 1, No. 2, 2018, pp. 74-78. DOI 10.26739/2523-1243

\section{dol http://dx.doi.org/10.26739/2523-1243/-2018-1-2-12}

\begin{abstract}
T-cell acute lymphoblastic leukemia (T-ALL) ranges from $10 \%$ to $15 \%$ of newly diagnosed cases of acute lymphoblastic leukemia (ALL) in children. The article analyzes the results of therapy of 31 patients with T-ALL who received treatment in the children's department of the clinic of the Research Institute of Hematology and Blood Transfusion (Uzbekistan, Tashkent) from June of 2009 to December 2014 according to the ALL-MB-2008 protocol.

In the study, the 3-year event-free survival (EFS) was $64.5 \%$. Low survival rates were recorded in patients with a high initial leukocyte count $(>100.0)$, large spleen size $(4 \mathrm{~cm}$ or more), poor early response to therapy ( patients with $30 \%$ or more blast cells in the bone marrow on the 15th day of induction). Coexpression of myeloid antigens CD13 and CD33 can also be attributed to factors of unfavorable prognosis.
\end{abstract}

Keywords: acute lymphoblastic leukemia, children, immunophenotyping, T-ALL, chemotherapy.

\section{Introduction:}

T-cell acute lymphoblastic leukemia (TALL) is from $10 \%$ to $15 \%$ of newly diagnosed cases of acute lymphoblastic leukemia (ALL) in children. Historically, patients with T-ALL have a worse prognosis compared to other types of ALL $[1,2]$.

The forecast of this option ALL is gradually improving with the advent of intensive chemotherapy (HT): the use of modern therapeutic protocols 10-year survival (EFS) of children with T-ALL as high as $75 \%$. Isolation of patients with T-ALL and in a separate subgroup analysis of prognostic factors and the effectiveness of various therapeutic options are justified in terms of the biological differences between this version ALL $[3,4,5]$.

Aim: The purpose of the analysis - 
assessment of the effectiveness of therapy and prognostic value of various factors in children with T-ALL during the treatment protocol ALL-MB-2008.

Material and methods: ALL-MB-2008 protocol is designed for the treatment of children with primary acute lymphoblastic leukemia at the age from 1 year to 15 years. The study was conducted from 2008. to December 2014 .. In total, the study involved 49 centers of Russia and Belarus, 4142 registered patients.

Children's department of Scientific Research of Hematology and Blood Transfusion the Ministry of Health of Uzbekistan (Tashkent) participated in the ALL-MB-2008 study from mid-2009 to December 2014. as the observation group, registered 300 patients with ALL.

The results of treatment of 31 patients on the protocol ALL-MB-2008 (ALL-
MB-2008) with T-ALL who received treatment at the clinic and the Research Institute Mr. PC (Uzbekistan, Tashkent) for the period 2009-2015. The diagnosis was based on clinical findings, myelogram, hemogram, immunological and molecular genetic analyzes. Immunological studies were performed by flow cytometry in cytological laboratory of the Russian Children's Clinical Hospital, molecular genetic studies in the laboratory of biochip diagnostics of Federal Research Center of Pediatric Hematology Oncology Immunology (Moscow).

All patients received chemotherapy according to ImRG, including a weekly intramuscular injection LD-MTX and asparaginase, additional intrathecal administration of three cytotoxic drugs. (Figure 1)

Fig.1. The protocol ALL-MB-2008

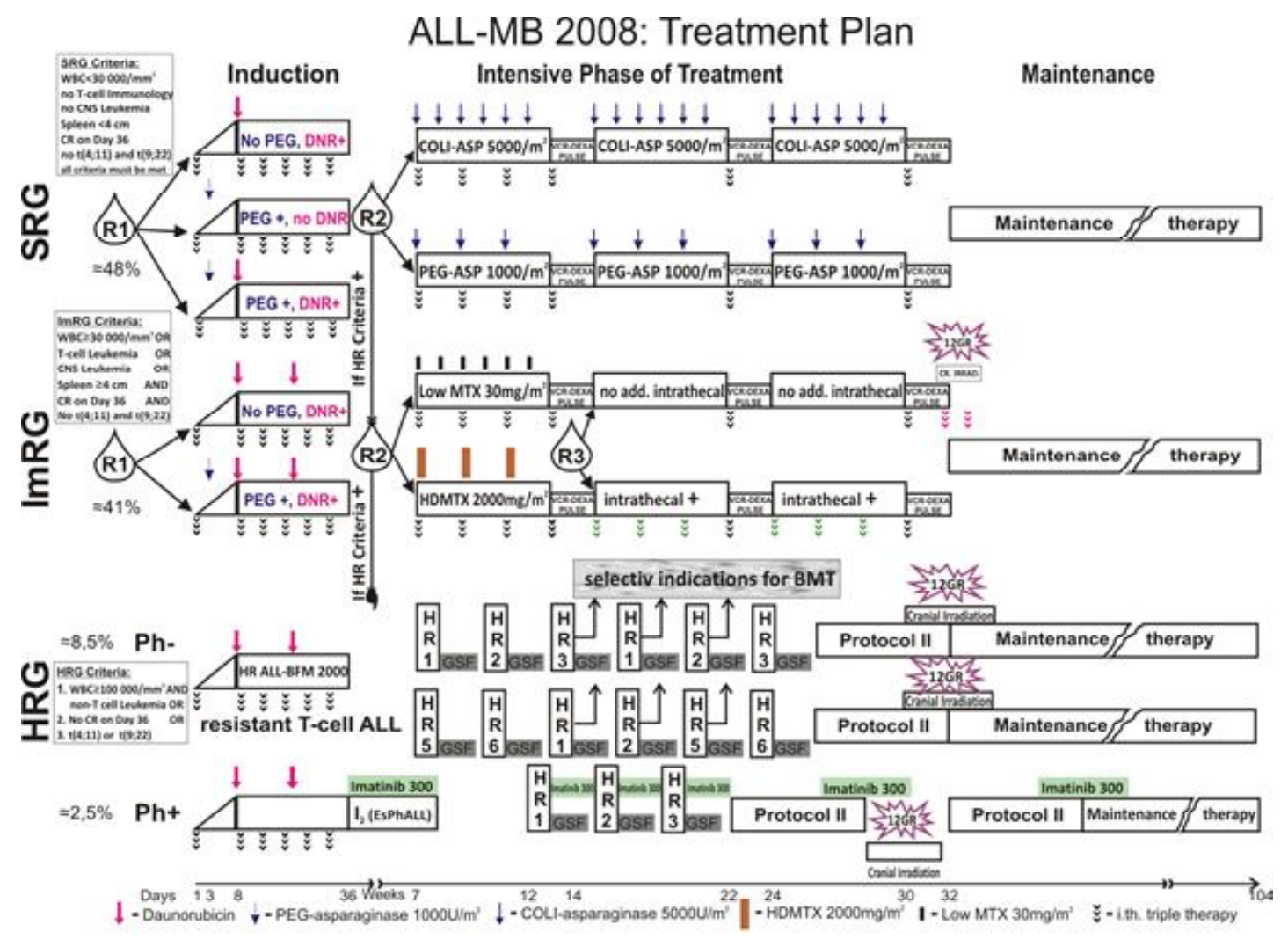


Bakhramov Saidjalol Makhmudovich, Ibragimova Sapura Zakhidovna. T-cell acute lymphoblastic leukemia in children: the experience of the treatment protocol ALL-MB-2008 in Uzbekistan.

Among patients with T-ALL was dominated by boys of $25(80.6 \%)$. Most of the patients were children from 5 to 10 years $-51.6 \%$, of children between 10 and 15 years was $38.7 \%$. 10 patients (32.2\%) at the time of diagnosis had the number of peripheral blood leukocytes less 30x109/1, $12(38.7 \%)$ patients had more than $100 \times 109 / 1$. Depending on the size of the spleen was as patients about the same: $58.1 \%$ of patients in the spleen appeared less than $4 \mathrm{~cm}$ from the edge of the costal arch, in $41.9 \%$ of patients $-4 \mathrm{~cm}$ or more. The defeat of the mediastinum were detected in 12 (38.7\%) patients, initial neuroleukemia not found a single patient. Immunological version of the T-ALL is defined according to the classification of the
European Group on immunological characterization of leukemia (European Group for the Immunological Charakterization of Leukemias - EGIL). Depending on the immunophenotype of patients were as follows: TI / TII-ALL option has been installed in $7(22.5 \%)$ patients, TIII-ALL option - in 18 (58.1\%) patients, TIV-option ALL - 6 ( $19.4 \%)$ patients. CD1a detected in 14 (45.1\%) patients. Coexpression of CD 13 was detected in $2(6.4 \%)$ patients, co-expression of CD 33 - in one patient. Only one patient was found chromosomal translocation $\mathrm{t}(6 ; 11)$ MLL / AF6, the remaining patients the chromosomal changes were not found. An initial characteristic of the patients are presented in Table. 1.

Table. 1. Initial characteristic of the patients.

\begin{tabular}{|l|l|l|}
\hline Factor & $\begin{array}{l}\text { Abs. number } \\
\text { of patients }\end{array}$ & $\%$ \\
\hline Sex: & 25 & 80,6 \\
Male & 6 & 19,4 \\
Female & 3 & \\
\hline Age, years: & 16 & 9,6 \\
Up to 5 years & 12 & 51,6 \\
$5-10$ & 10 & 38,7 \\
$10-15$ & 9 & 32,2 \\
\hline An initial white blood cell count, x10 / 1: & 29,1 \\
Less than 30.0 & 12 & 38,7 \\
$30,0-100,0$ & 18 & \\
Eoлee 100,0 & 13 & 58,1 \\
\hline The size of the spleen, cm: & & 41,9 \\
Less than 4.0 & 7 & \\
4,0 or more & 18 & 22,5 \\
\hline immunophenotype: & 6 & 58,1 \\
TI/TII- variant & & 19,4 \\
TIII- variant & 12 & 38,7 \\
TIV- variant & 19 & 61,3 \\
\hline The defeat of the mediastinum & & \\
Yes & - & 100 \\
No & 31 & \\
\hline CNS status & & \\
III & & \\
I/II & & \\
\hline & & \\
\hline
\end{tabular}




\section{Results:}

After the induction course went into remission 27 (87\%) patients. Out of 31 patients in the induction of death pronounced in 2 patients (6.4\%). In 3 of remission of patients failed to achieve, 1 died of progression of the disease, two of the patients consolidation by the intermediate-risk group was continued with low-dose chemotherapy in the absence of conditions for therapy for $\mathrm{HRG}$ patients achieved remission: one complete remission duration remains, another 1.5 years ascertained relapse. Lost to follow-up (LFU) - 1 patient $(3.2 \%)$. In $6(19.4 \%)$ patients had relapsed: one patient isolated CNS relapse, others had bone marrow relapse. The median was 1 year. 3 -year diseasefree survival (EFS) was $64.5 \%$. Treatment results are presented in table 2.

Table 2. The results of treatment of patients with T-ALL

\begin{tabular}{|l|l|l|}
\hline Factor & $\begin{array}{l}\text { Abs. number } \\
\text { of patients }\end{array}$ & $\%$ \\
\hline Death in Induction & 2 & 6,4 \\
\hline Non-responder & 3 & 9,6 \\
\hline Complete remission & 25 & 80,6 \\
\hline Relapse: & & \\
bone marrow & 5 & 16,1 \\
Isolated CNS relapse & 1 & 3,2 \\
\hline Death in remission & 2 & 6,4 \\
\hline LFU & 1 & 3,2 \\
\hline CCR & 20 & 64,5 \\
\hline
\end{tabular}

The greatest number of relapses were observed in patients with spleen size greater than $4 \mathrm{~cm}$ and an initial leukocytosis more than $100 \times 109 / 1$. There were 13 patients (41.9\%). 4 patients relapsed ascertained, one is death in the induction, one - death in remission. Relapse-free survival (EFS) in this group of patients was $53.8 \%$ compared with $77.7 \%$ in the spleen size of less than $4 \mathrm{~cm}$ and less than an initial leukocytosis $100 \mathrm{~h} 109 / \mathrm{l}$. In the Russian-Belarusian group EFS 75,6\% in this patient group compared with $87 \%$ in the spleen size of less than $4 \mathrm{~cm}$ and less than an initial leukocytosis 100x109/ 1 .

Poor response to induction ascertained day 15 in $10(32.2 \%)$ patients. In $3(30 \%)$ patients relapse ascertained, one (10\%) - death induction, 2 patients - no remission on day 36. Relapse-free survival (EFS) in this group of patients was $30 \%$.

Patients expressing CD1a + was 21 $(67.7 \%)$. In 4 (19\%) patients relapse ascertained, one - death in remission, one - no remission on day 36 .

In 3 patients revealed coexpression of CD13 and CD33 myeloid antigens, all ascertained relapse. One patient revealed a translocation t $(6 ; 11)$ MLL / AF6, poor response on day 15 ( $83 \%$ blasts), in a year in this patient developed bone marrow relapse.

Conclusion: Thus, at the moment it is clear, that patients with T-ALL were 
Bakhramov Saidjalol Makhmudovich, Ibragimova Sapura Zakhidovna. T-cell acute lymphoblastic leukemia in children: the experience of the treatment protocol ALL-MB-2008 in Uzbekistan.

heterogeneous group. In the study, the 3year event-free survival (EFS) was $64.5 \%$. Low survival rates were recorded in patients with a high initial leukocyte count $(>100.0)$, large spleen size $(4 \mathrm{~cm}$ or more), poor early response to therapy ( patients with $30 \%$ or more blast cells in the bone marrow on the 15th day of induction). Coexpression of myeloid antigens CD13 and CD33 can also be attributed to factors of unfavorable prognosis.

\section{Literature}

1. Rumyantsev AG, Samochatova EV, Hamdan T. Terapiya acute lymphoblastic leukemia in children BFM program. Pediatrics. 1991; 11: 58-63.

2. Karachunsky AI, Rumyantsev AG, Stackelberg A., et al. Comparison of protocol ALL-BFM-90 and ALL-MB-91 for the treatment of acute lymphoblastic leukemia in children (pre D results). Pediatrics. 1995; $2: 10-14$.

3. Karachunsky AI Myakov NV, Y. Rumyantsev, etc. The results of a multicenter trial of acute lymphoblastic leukemia in children program ALL-MB-91 / ALL-BFM-90m:. Analysis of efficacy and toxicity. Ter. archive. 2007; 79 (7): 19-26.

4. Karachunskiy A, Herold R, von Stackelberg A et al.Results of the first randomized multicentre trial on childhood acute lymphoblastic leukaemia in Russia. Leukemia. 2008; 22 (6): 1144-1153/

5. Goldberg JM1, Silverman LB, Levy DE, Dalton VK, Gelber RD, Lehmann L, Cohen HJ, Sallan SE, Asselin BL. J Clin Oncol. 2003 Oct 1; 21(19):3616-22. Childhood T-cell acute lymphoblastic leukemia: the Dana-Farber Cancer Institute acute lymphoblastic leukemia consortium experience. 